\title{
Sobre una nueva inscripción latina funeraria en Lusitania
}

\author{
On a new funerary roman inscription in Lusitania
}

\author{
Javier Andreu Pintado - Javier Ramírez García*
}

\begin{abstract}
RESUMEN
ABSTRACT

El objetivo del presente trabajo es el de dar a conocer una nueva inscripción romana, funeraria, sobre granito, procedente del término municipal de Don Benito (Badajoz) y seguramente adscrita al tipo de estelas graníticas tan habituales en el paisaje funerario de Augusta Emerita y su entorno. La pieza -muy fragmentaria- atestigua pese a ello la presencia de un nuevo antropónimo, Surina, hasta ahora no constatado en la epigrafía peninsular. PALABRAS CLAVE

Epigrafía, Augusta Emerita, inscripciones funerarias, estelas graníticas, onomástica, Surinus, Lusitania.

The aim of this paper is to present a new roman inscription, a funerary one, carved on granite and founded in the municipality of Don Benito (Badajoz) probably related with the usual type of granite funerary stelae commonly attested in the funerary landscape of Augusta Emerita. Instead of the very fragmentary aspect of the piece it certifies the presence of a new female name, Surina, still unattested in the hispanic epigraphy.

\section{KEYWORDS}

Epigraphy, Augusta Emerita, funerary inscriptions, granite stelae, onomastics, Surina, Lusitania.

Recibido el 19 de mayo de 2012. Aceptado el 5 de agosto de 2012

Seguramente junto con la de Tarraco y su entorno, la documentación epigráfica de Augusta Emerita y su área de influencia inmediata — si se quiere todo el antiguo conuentus Emeritensis - conforma uno de los repertorios documentales más sugerentes de la epigrafía hispánica. Con todo, pese a que —cuantitativa, por

\footnotetext{
* Javier Andreu Pintado, Profesor Titular de Historia Antigua, Universidad Nacional de Educación a Distancia, Senda del Rey, 7, 5aㅗ planta, 28040 Madrid: jandreu@geo.uned.es. Javier Ramírez García, estudiante de Tercer Ciclo del Departamento de Historia Antigua de la Universidad Nacional de Educación a Distancia, Plaza de España, 1, 06410 Santa Amalia (Badajoz): javiramirez@ hotmail.es. El presente trabajo se ha visto enriquecido por las aportaciones de los informantes y evaluadores externos de la Serie 2 de Espacio, Tiempo y Forma que, aquí, quieren agradecerse siendo, en cualquier caso, los errores y omisiones que el lector pueda percibir responsabilidad última y absoluta de sus firmantes.
} 
el número de inscripciones conservadas, y cualitativamente, por la riqueza histórica de muchas de ellas- ello es innegable, no es menos cierto que la investigación -necesariamente epigráfica- sobre la sociedad Emeritense y sobre la de ese ámbito geográfico concreto de la Lusitania romana se ha encontrado siempre notablemente mediatizada por varios condicionantes de naturaleza, si se quiere, metodológica o, cuando menos, instrumental o documental. Por un lado, el principal corpus epigráfico sobre la colonia, sobre Augusta Emerita, permanece todavía inédito $^{1}$ y, por otro, ni Cáceres ni Badajoz cuentan en la práctica con repertorios epigráficos completos ${ }^{2} \mathrm{y}$, cuando los tienen - como en el caso de Cáceres- éstos se hallan totalmente desfasados o están aun en feliz proceso de actualización ${ }^{3}$. Ese obstáculo metodológico y heurístico, sin embargo, la investigación lo está supliendo con nuevos y muy meritorios esfuerzos hermenéuticos como los que, en los últimos años, bien han presentado científicamente nuevos tituli de naturaleza pública y de interés totalmente inusitado ${ }^{4}$, bien se han esforzado por reorganizar parte del material disponible ${ }^{5}$ o han empezado a constatar nuevos documentos en áreas hasta hace poco casi de vacío documental ${ }^{6}$. Ya hace algunos años, de hecho, que un conocido investigador sobre la sociedad de la Augusta Emerita altoimperial señalaba que sólo la publicación de tantas inscripciones como están, todavía -en Mérida y en otros municipios del ámbito extremeño - en manos de

1 GARCÍA IGLESIAS, Luis, Epigrafía romana de Augusta Emérita, (Tesis Doctoral, s. p.), Universidad Complutense, 1973.

2 Para Badajoz véase, en todo caso, SALAS MARTín, José, Catálogo de las inscripciones romanas y cristianas del Museo Arqueológico Provincial de Badajoz. Badajoz, Regional de Extremadura, 1997 y, para su área meridional, perteneciente a la antigua Beturia, el trabajo de referencia de CANTO, Alicia Mํ.., Epigrafía Romana de la Beturia Céltica. Madrid, Universidad Autónoma, 1997, pp. 47-146 además de CIL II/7, 851-1014, pp. 193-230 aunque en la Antigüedad este territorio deba adscribirse al ámbito bético.

${ }^{3}$ HURTADO DE SAN ANTONIO, Ricardo, Catálogo provincial de inscripciones latinas. Cáceres, Diputación Provincial, 1977 y ESTEBAN ORTEGA, Julio, Corpus de Inscripciones de Cáceres. I. Norba. Cáceres, Junta de Extremadura, 2007 como primer paso de la referida actualización.

${ }^{4}$ Por ejemplo HEp13, 127, del foro provincial de la colonia capital: STYLOW, Armin y VENTURA, Ángel, "Los hallazgos epigráficos", en AYERBE, Rocío, PALMA, Félix y BARRIENTOS, Teresa (coords.), El foro de Augusta Emerita. Génesis y evolución de sus recintos monumentales. Madrid, Anejos de AEspA, 2009, pp. 455-457, ㄲo 1.

${ }^{5}$ Para el caso concreto de Augusta Emerita resultan ejemplares RAMíREZ SÁDABA, José Luis y MATEOS, Pedro, Catálogo de las inscripciones cristianas de Mérida. Mérida, Museo Nacional de Arte Romano, 2000; RAMÍREZ SÁDABA, José Luis, Catálogo de las inscripciones imperiales de Augusta Emerita. Mérida, Museo Nacional de Arte Romano, 2003; EDMONSON, Jonathan, Granite funerary stelae from Augusta Emerita. Mérida, Ministerio de Cultura, 2006 o NOGALES, Trinidad, RAMÍREZ SÁDABA, José Luis y MURCIANO, José Mํ.., "Las cupae del territorium Emeritense", en ANDREU, Javier (ed.), Las cupae hispanas. Origen, difusión, uso, tipología. Uncastillo, Fundación Uncastillo/UNED de Tudela, 2012, pp. 349-368 (este volumen también incluye novedades singulares respecto de Cáceres y del reborde meridional de la provincia de Badajoz: pp. 415-434 y pp. 221-270 respectivamente).

${ }^{6}$ Singular en este sentido es el caso del entorno de la región de la Vera, en Cáceres, donde no son pocas, anualmente, las novedades epigráficas (véase un balance, al menos, en GÓMEZ-PANTOJA, Joaquín, MADRUGA, José-Vidal, y GONZÁLEZ CORDERO, Antonio, “¿Un raro tipo de monumento sepulcral?", en ANDREU, Javier, ESPINOSA, David y PASTOR, Simone (eds.), Mors omnibus instat. Aspectos arqueológicos, epigráficos y rituales de la muerte en el Occidente Romano. Madrid, Liceus E-Excellence/UNED de Tudela, 2011, pp. 383-406, con bibliografía). 
particulares y que, por tanto, permanecen inéditas, podría, si quiera, arrojar algo más de luz sobre las incógnitas que el conocimiento de los procesos históricos de la zona en la Antigüedad, estos condicionantes documentales, y la propia práctica investigadora en Ciencias de la Antigüedad, han ido planteando en los últimos años ${ }^{7}$. Ése es precisamente el objetivo de estas páginas, dar a conocer una nueva inscripción del ámbito de influencia de la colonia capital de Lusitania que, más allá de su interés intrínseco — ciertamente limitado por el estado fragmentario de la pieza-, aporta un antropónimo hasta ahora no atestiguado en el ámbito hispano.

En el invierno del curso 2000-2001, en el contexto de los trabajos de explanación y desmonte de una alameda en la finca de "Los Ventosos" próxima al pueblo de colonización Hernán Cortés — término municipal de Don Benito (Badajoz) - y en las proximidades del curso del río Ruecas, junto a otra pieza —en paradero desconocido, seguramente enterrada de nuevo por ulteriores labores de remoción de tierra- que, a tenor de las explicaciones facilitadas por el descubridor de ambas, D. Luis Sánchez Gállego, debía contar con un crismón y con un pez como principales motivos decorativos - seguramente, por tanto, una placa o estela funeraria paleocristiana - fue hallado un fragmento granítico de tonos rojizos, de $38 \mathrm{~cm}$ de ancho, $14 \mathrm{~cm}$ de grosor en su base y $25 \mathrm{~cm}$ de altura, perteneciente al ángulo izquierdo de una inscripción que, por su fábrica y características, pudo formar parte de una estela funeraria como las que están bien documentadas en el repertorio epigráfico Emeritense ${ }^{8}$ por más que el lugar del hallazgo —el actual término municipal de Don Benito- se haya vinculado al territorium de la colonia de Metelli$n u^{9}$ si bien es conocido el debate que existe sobre la conciliación de los límites del generosísimo territorium de Augusta Emerita con los del de la colonia Metelli-

7 SAQUETE, José Carlos, Las elites sociales de Augusta Emerita. Mérida, Museo Nacional de Arte Romano, 1997, p. 17.

${ }^{8}$ EDMONSON, Jonathan, op. Cit. (n. 5). El estado fragmentario de la pieza (Figs. 1 y 2) no impide descartar por completo la posibilidad de que se tratase de un bloque arquitectónico -cierto que poco probable dada la escasa envergadura del fondo de la misma- o de una placa como también consta que se fabricaron éstas en granito en la zona en época romana (véase, por ejemplo, EDMONSON, Jonathan, op. Cit. (n. 5), pp. 205-218) no en vano la parte trasera presenta una cierta concavidad, tal vez pensada para la fijación del soporte a un conjunto mayor si no es que dicha concavidad es secundaria o fruto del deterioro del soporte. A favor de su interpretación como estela, en cualquier caso, están sus dimensiones -que, como se verá, encajan en la metrología habitual de los tipos de estelas graníticas atestiguados en Augusta Emerita-, el hecho de que la parte trasera haya sido también trabajada y pulida (un sinsentido si la pieza hubiera sido pensada para su encastre en un monumento mayor), la apretada composición de la fórmula epigráfica que ocupa la línea primera -que permite pensar en que el lapicida no disponía de mucho espacio más a la derecha-y, por supuesto, el hecho de que este tipo de soporte fuera de los más habituales en las inscripciones funerarias de la zona.

9 Véase FERNÁNDEZ CORRALES, José M., ALVARADO, Manuel, ÁlVAREZ MARTíNEZ, José Mª y VELÁZQUEZ, Agustín, "Metellinum", en Tabula Imperii Romani. Hoja J-30: Valencia. Corduba. Hispalis. Carthago Noua. Astigi. Madrid, Ministerio de Fomento, pp. 235-236 o GONZÁLEZ CORDERO, Antonio; SUÁREZ DE VENEGAS, José y ALVARADO, Manuel de, "Nuevas aportaciones a la Epigrafía de Extremadura", Alcántara, 21, (1990), pp. 113-150, con las tres únicas inscripciones publicadas, hasta la fecha, como procedentes de dicho término municipal: $H E p 4,144=H E p 10,54$ e $H E p 4,145$ y $146=A E$ $1997,791$. 
nense $^{10}$ y sobre la adscripción o no de algunos términos municipales actuales ubicados al Oeste de Mérida a ese antiguo y vastísimo territorio de la vieja capital provincial ${ }^{11}$. Según las noticias transmitidas por los vecinos del lugar y confirmadas por el descubridor de la pieza, junto a la inscripción que aquí nos ocupa y junto a la placa —al parecer también granítica - con motivos paleocristianos antes aludida aparecieron vasijas, huesos y materiales varios que permiten pensar que el contexto de hallazgo de la inscripción en cuestión es el de una antigua necrópolis, tal vez asociada a alguna unidad de poblamiento rural aun por determinar.

La inscripción - que hoy se conserva en Don Benito, en propiedad de su descubridor, quien, gentilmente, nos facilitó la realización de su autopsia y del correspondiente reportaje fotográfico en Mayo de 2012 - presenta tres líneas de texto grabadas en capitales cuadradas de carácter rústico muy limitadas en su factura - especialmente en los trazos más diagonales de la $\mathrm{V}$ o de la $\mathrm{N}$ lo que ha originado, por ejemplo, la apenas imperceptibilidad de la $\mathrm{N}$ final de la tercera líneapor los condicionantes propios del material empleado como soporte, el granito ${ }^{12}$; letras que presentan un módulo de $6,5 / 7 \mathrm{~cm}$. Sólo en la primera línea es perceptible una interpunción clara separando las dos fórmulas funerarias, de carácter circular (Fig. 1) aunque existe una menor, centrada respecto de las líneas, conforme se in-

10 Como reproducir aquí de nuevo el debate sobre los límites del territorium Emeritense -especialmente centrado, en cualquier caso, en el ámbito meridional y en el occidental a partir de, al menos WIEGELS, Rainer, "Zum Territorium der Augusteische kolonie Emerita", Madrider Mitteilungen, 17, (1976), pp. 258-284 y CANTO, Alicia Ma.., "Colonia lulia Augusta Emerita: consideraciones en torno a su fundación y territorio", Gerión, 7, (1989), pp. 149-206, aunque existen más trabajos- excede los límites de esta noticia epigráfica, remitimos para su seguimiento a SÁNCHEZ BARRERO, Pedro D., "Territorio y sociedad en Augusta Emerita”, en GORGES, Jéan-Gerard y NOGALES, Trinidad (eds.), Sociedad y cultura en Lusitania romana. IV Mesa Redonda Internacional. Mérida, Junta de Extremadura, 2000, pp. 203-225, esp. pp. 218-219 donde se aborda -con toda la bibliografía- la problemática del límite oriental, aun no zanjada además de a CORDERO RUIZ, Tomás, "Una nueva propuesta sobre los límites del ager Emeritensis durante el Imperio Romano y la Antigüedad Tardía”, Zephyrus, 65, (2010), pp. 149-165. La pieza aquí presentada, desde el punto de vista de su soporte, tampoco permite esclarecer el asunto pues si el granito es material clave en la producción epigráfica y escultórica emeritense (NOGALES, Trinidad, "Las estelas funerarias peninsulares en el mundo clásico. El fenómeno Emeritense”, en DE LA CASA, Carlos (ed.), Actas del V Congreso Internacional de Estelas Funerarias. Soria, Diputación de Soria, 1994, pp. 201210), no lo es menos en la de Metellinum donde también se conoce un buen número de estelas labradas en este material (CIL II, 611; AE 1987, 487; AE 1997, 793 o HEp8, 18, véase HABA, Salvadora, Medellín romano. La colonia Metellinensis y su entorno. Badajoz, Diputación de Badajoz, 1998, pp. 89-90 y 149152). Acaso sí la paginación y algunos aspectos internos del texto parecen remitir más a paralelos Emeritenses que a paralelos Metellinenses por más que en este sentido el hecho de que el repertorio epigráfico de Augusta Emerita sea mayor y el número de elementos de comparación disponibles sea, por ello, más generoso, haya de ser tomado en consideración.

11 El debate y la bibliografía puede seguirse también a partir de OSLAND, Daniel, The Early Roman Cities of Lusitania. Oxford, British Archaeological Reports, 2006, pp. 50-51.

12 Sobre estos problemas paleográficos y técnicos de las inscripciones graníticas Emeritenses puede verse, de nuevo, EDMONSON, Jonathan, op. Cit. (n. 5), pp. 80-81 además de las advertencias hechas en su día -en un capítulo metodológicamente inexcusable- por SAQUETE, José Carlos, op. Cit. ( $\mathrm{n}$. 7), pp. 18-19 haciéndose eco, además, de una limitación técnica -y, para los epigrafistas, también cronológica y de datación- ya advertida por GARCÍA IGLESIAS, Luis, op. Cit. (n. 1), p. 13 respecto de la documentación epigráfica de Augusta Emerita. 
dica más adelante. El texto conservado (Fig. 2), sin mayores problemas de lectura excepto en el final del tercer renglón, donde el trazo de la $\mathrm{N}$-que parece existir y así se ha comprobado en la autopsia - aparece mucho menos marcado que el correspondiente al resto del epígrafe, podría decir así:

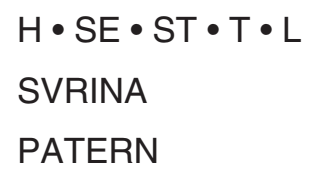

PATERN

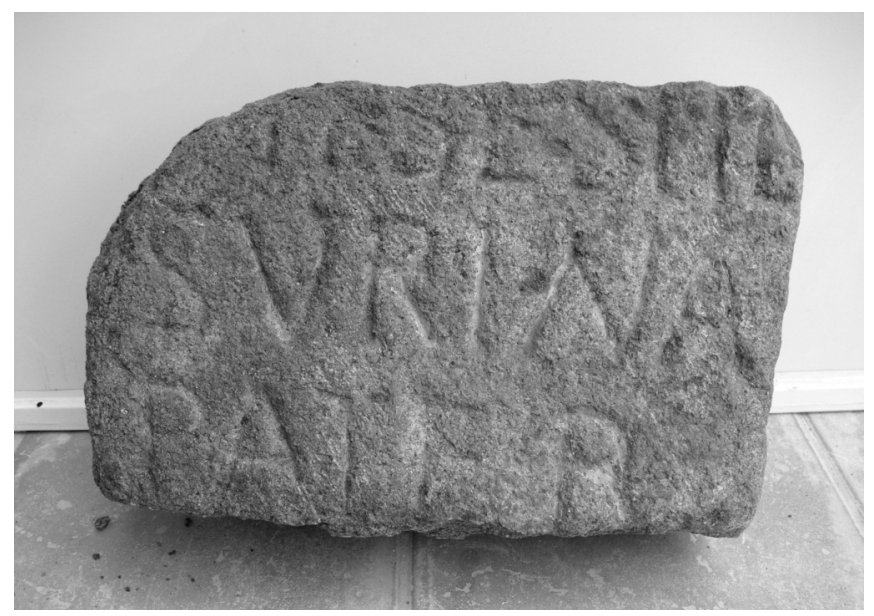

Fig. 1: Aspecto general de la inscripción de Don Benito (Foto: J. Ramírez).

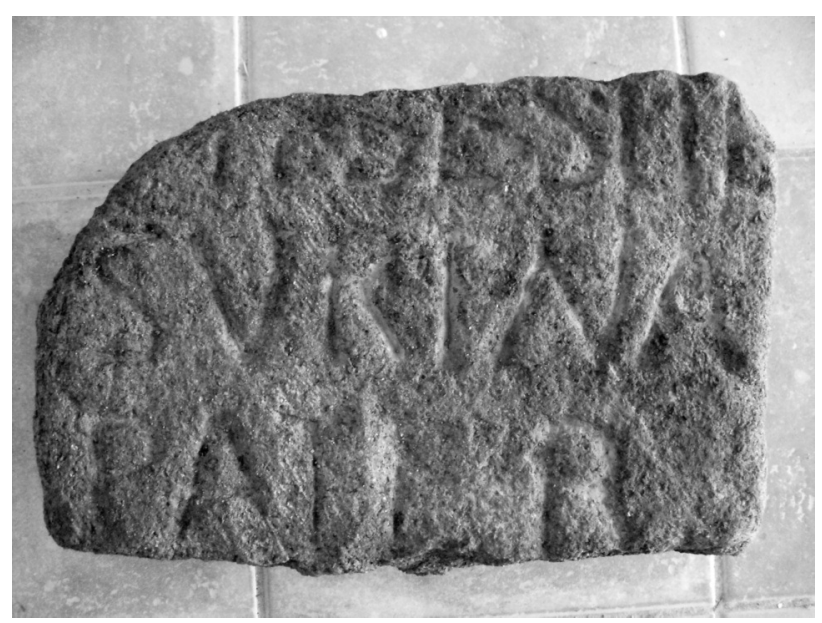

Fig. 2: Detalle del texto del epitafio hallado en Don Benito (Foto: J. Ramírez). 
La disposición de los elementos, la paginación del texto, el tipo de soporte sobre el que — presumiblemente- está realizada y, por supuesto, la ordinatio habitual de este tipo de epitafios permiten pensar que disponemos de más de la mitad de la estela -los 40/42 cm son una de las medidas medias habituales para el ancho de este tipo de soportes producidos en los talleres de Augusta Emerita ${ }^{13}$ (recuérdese que conservamos $38 \mathrm{~cm}$ ) mientras que en altura, salvo raras excepciones, no pasan de los $60 \mathrm{~cm}-$ y que, por tanto, para el texto en cuestión, habría que pensar en una restitución semejante a la que se ofrecerá más abajo. En ella $-y$ teniendo en cuenta que pueden faltarnos cuando menos unos 20/25 cm de pieza en altura- se podrían suponer, al menos, tres líneas iniciales, una para la inserción de la fórmula epigráfica funeraria habitual, $D$ (is) M(anibus) s(acrum), otras dos para la presentación del nombre del difunto y, acaso, una más para la indicación de su edad, y, a partir de ahí, las tres líneas de texto que conservamos, muy dañada, como es evidente (Fig. 2), la primera, pero de lectura perfectamente restituible. Es en la segunda línea en la que constatamos la verdadera novedad de la inscripción, la presencia de un antropónimo, Surina, hasta ahora no atestiguado en la Península Ibérica y, por tanto, del máximo interés.

Se trata, Surina -y su variante masculina, Surinus - de un antropónimo atestiguado fuera de Hispania tanto como gentilicio como - muy especialmente- en calidad de cognomen ${ }^{14}$ pero no presente, como se ha dicho, en la Península Ibérica $^{15}$. Así, como gentilicio se conoce a un M(arcus) Surinus adscrito a la Pupinia tri-

13 Véase, por ejemplo, EDMONSON, Jonathan, op. Cit. (n. 5), nos 3 (=HEp6, 123), no 8, no 10 (HEp6,

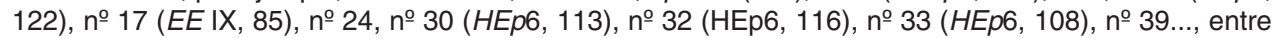
otras.

14 MÓCSY, Andreas, FELDMANN, Reinhard, MARTON, Elizabeth y SCILÁGYI, Maria, Onomasticon provinciarum Europeae Latinarum (OPEL). Vol. IV. Quadratia-Zures. Viena/Budapest, Archaeolingua, 2002, p. 101. Un inventario de casos puede consultarse también en KRAHE, Hans, Lexikon altillyrischer Personennamen. Heidelberg, Winter, 1929, pp. 108-109.

15 ABASCAL, José Manuel, Los nombres personales en las inscripciones latinas de Hispania. Murcia, Universidad de Murcia, 1994. Desde BOLDETTI, Marco A., Osservazioni sopra i cimiteri de santi martiri ed antichi cristiani di Roma. Roma, Archiginnasio della Sapienza, 1720, p. 55 y desde DE ROSSI, Juan Bautista y FERRUA, Antonio, Inscriptiones Christianae Vrbis Romae. VI. Roma, Befanus, 1975, p. 236 (a propósito de ICUR VI, 16992) y SEPPARD, Frere, Roman Inscriptions of Britain. Volume II. Fascicule 6. Oxford, Clarendon Press, p. 28 se viene considerando el cognomen Surinus como un derivado del cognomen Sura que KAJANTO, liro, The Latin Cognomina. Helsinki, Societas Scientiarum Fennica, 1965, p. 226 (y a partir de él también ABASCAL, José Manuel, op. Cit., p. 518) incluyó entre los cognomina vinculados a partes del cuerpo -sura="pantorrilla"- y que en la epigrafía peninsular está bien atestiguado en el repertorio epigráfico de la colonia de Barcino en la colección relativa a L. Licinius Sura (IRC IV, 83 a 104) y que, en su versión masculina, Surus, está bien presente en Lusitania (véase NAVARRO, Milagros (coord.), Atlas antroponímico de la Lusitania Romana. Mérida/Burdeos, Fundación de Estudios Romanos/Ausonius, 2003, pp. 308-309, Mapa 284) en inscripciones de Ávila (LICS 48), Idanhãa-Velha (ILER 973), Villamesías (CPILC 618), Mérida (ILER 4606) -aunque en este caso en la variante Syra, con aquella relacionada (LOZANO, Arminda, Die griechischen Personnennamen auf der iberischen Halbinsel. Heidelberg, Winter, 1998, pp. 230-231) - y Vila Viçosa (IRCP 441). Varios han sido los autores (especialmente siguiendo a ALBERTOS, Mª Lourdes, La onomástica personal primitiva de Hispania Tarraconense y Bética. Salamanca, Instituto Antonio de Nebriga, 1966, p. 214 pero también a KNAPP, Robert C., Latin Inscriptions of Central Spain. Berkeley/Los Ángeles/Oxford, University of California Press, 1992, pp. 47 y 48) que han considerado que el origen de dicho cognomen o, al menos, su éxito en ám- 
bus en una inscripción de la Isola Sacra (CIL V, 483) y a un Marcus Surinus Marcellus magistrado municipal de Tergeste $(C I L ~ V, 544)^{16}$ mientras que, en la variante femenina, Surina, exclusivamente lo encontramos como cognomen bien en estructuras onomásticas bimembres - como Aurelia Surina, en una inscripción de la necrópolis paleocristiana de la vía Labicana de Roma (ICUR VI, 16992)— bien en otras más simples como la Surina esposa de Virilis que aparece en un grafito sobre cerámica hallado en Alcester, Warwickshire, en el Reino Unido (RIB II, 6, 2493. 54) o la Surina hija de Gaudentius a la que se alude en otra inscripción cristiana de la necrópolis romana de la vía Tiburtina (ICUR VII, 19387). Aunque la alta concurrencia de este gentilicio - y también de las atestiguaciones de Surina como cognomen- en la epigrafía itálica podrían ponernos tras la pista de las lógicas conexiones itálicas de las colonias romanas de Augusta Emerita o de Metellinum -por otra parte bien atestiguadas y esperables para dos fundaciones de cronología augústea y cesariana respectivamente ${ }^{17}$ añadiendo, si cabe, un atractivo histórico al monumento aquí presentado - nos parece que el contexto 'provincial' al que debe adscribirse la inscripción que nos ocupa nos debe llevar a rechazar esa opción interpretativa desechando la opción de lectura Surina / Patern[a] — gentilicio y cognomen - y permitiendo, en cambio, pensar en que - como por otra parte hemos visto que resulta habitual en otros testimonios onomásticos conservados en ámbitos de fuerte raigambre céltica en la Península- Surina aparece en nuestra inscripción como cognomen y no como gentilicio de ahí que propongamos una lectura Surina [et] Patern[a] y no Surina Paterna pues entendemos que, por la prolongación del renglón inicial del texto podría, a la derecha de lo conservado - Surina - haber espacio, para, al menos, un término más, no superior a dos caracteres: et. Lamentablemente, esto no puede confirmarse en el estado actual de conservación de la pieza aunque parezca la interpretación más probable a nuestro juicio.

Con todo lo dicho, la formación de Surina como antropónimo podría, pues, conectarse, como se ha dicho más arriba, con algunas de las raíces indoeuropeas que han generado cognomina parecidos de una mayor atestiguación en la onomástica peninsular siguiendo una práctica onomástica que, como se dijo a pie

bitos de raigambre indígena -especialmente de cariz céltico- debería ponerse en relación con la raíz indoeuropea *Suro, que remite a los conceptos de "amargo" o "salado" (véase, por ejemplo, ENCARNAÇÃO, José d'., Inscrições romanas do conventus Pacensis. Coimbra, Faculdade de Letras, 1984, p. 526). Sea como fuere, bien como gentilicio, bien como cognomen, Surina es un hapax en la onomástica peninsular lo que añade interés al fragmento epigráfico aquí considerado por más que pueda relacionarse con cognomina bien atestiguados como los aquí citados o los muy próximos de Suria, que aparece referido en una inscripción de Salvatierra de Santiago, en Cáceres (CPILC 435), de Surianus, atestiguado en la propia Augusta Emerita (EE IX, 51), de Surisca, atestiguado en Talavera de la Reina (CIL II, 5339) o de Surilla, bien presente en Hispania en inscripciones de Belorado (AE 1985, 589) o de Tarraco (RIT 472 y 906), todos relacionables con esa raíz céltica *Suro a la que se aludió con anterioridad.

16 Véase, además, sobre éste, y sobre dicho gentilicio STICOTTI, Pedro (cur.), Inscriptiones Italiae. Volumen X-Regio X. Fasciculus IV-Tergeste. Roma, Libreria dello Stato, 1951, p. 35.

17 Véase, al respecto, SAQUETE, José Carlos, op. Cit. (n. 7), pp. 39-52 y 78-79 (con datos onomásticos en este último lugar) así como la síntesis de NAVARRO, Milagros (coord.), op. Cit. (n. 15), pp. 413-417. 
de página, casaría más con el tipo de inscripción ante el que nos encontramos. Qué duda cabe que, seguramente, si estuviésemos ante una inmigrante de origen itálico (Surina Paterna) muy probablemente se habría empleado un soporte epigráfico más noble como, de hecho, consta en gran parte de la epigrafía de la colonia ${ }^{18}$. Así las cosas, todo parece apuntar a que los dedicantes son dos individuos Surina y Patern[a] - lo ajustado, como antes se dijo, de la expresión, por parte del lapicida, de la fórmula inicial en la línea primera permite pensar que la inscripción apenas admitiría, como mucho, un par de letras más por renglón a la derecha- pudiéndonos encontrar ante un texto organizado del modo que figura más abajo y con un segundo nombre, Paterna - pues para la variante masculina, Paternus, no parece haber espacio, como se dijo- como segundo comitente, notablemente bien atestiguado en Lusitania $^{19}$ y sobre el que huelga incidir más aquí.

$$
\mathrm{h}(\text { ic) } \bullet \text { s(itus vel sita) e(st) } \bullet \mathrm{s}(\text { it) t(ibi) } \bullet \text { t(terra) } \bullet \text { I(euis) }
$$

\section{Surina [et] \\ Patern[a?]}

Esta estructura compositiva propuesta para el texto casa, de hecho, con la que revelan algunos epitafios de Augusta Emerita en los que la fórmula $h$ (ic) s(itus) e(st) s(it) t(ibi) t(erra) I(euis) aparece no cerrando el texto sino tras el nombre del difunto e inmediatamente antes que la mención de los comitentes como -por ejemplo y sin ánimo de exhaustividad-se aprecia en los casos del dedicado por Argentar(ia) Verana a su sobrino M(arcus) Argentarius Achaicus (HEp5, 89), el erigido por $P$ (ublius) Curtius Italicus a su padre $P$ (ublius) Curtius Cypaerus (HEp5, 93), el consagrado por Aemilius Pudens a su hijo Aemilius Pudentianus (CIL II, 488), el del retiarius Cassius Victorinus, dedicado por Antonia Seuera (AE 1962, 59), o el de Maryus Tichius consagrado a su padre Marius Drosus (CIL II, 577), entre otros muchos. En el ámbito de las estelas graníticas de la colonia también esta praxis de introducir el nombre del dedicante inmediatamente después de la fórmula deíctica y de la propiciatoria del descanso del difunto es habitual estando documentada, por ejemplo, en la inscripción que $A(e)$ qua dedica a su hija (H)eluia Paulla, en la que $C$. Licinius Sabinus erige en memoria de su esposa Paula Iulia, en la dedicada por Graphica a su marido lulius Longinus, en el epitafio de G(aius) Vpilius Asclepius o en el que Cominia Marcella dedica a su marido M(arcus) Valerius $\operatorname{Proc}(u) / u s^{20}$. Por último, tampoco faltan en la colonia casos en los que la fórmula aludida precede a dedicaciones funerarias protagonizadas por dos individuos juntos caso, por ejemplo, del epitafio de lul(ia) Glyconis promovido por sus hijos L(ucius) Munatius Asclepiades y M(arcus) Lucanius Auit(us) (AE 1993, 907), el de-

18 De nuevo, SAQUETE, José Carlos, op. Cit. (n. 7), pp. 104-114.

19 Véase, con el correspondiente expurgo, la lista facilitada por ABASCAL, José Manuel, op. Cit. (n. 15), pp. 449-451 y, lógicamente, la atención que le dedica el trabajo de NAVARRO, Milagros (coord.), op. Cit. (n. 15), p. 257, Mapa 224).

20 EDMONSON, Jonathan, op. Cit. (n. 5), nos 24 -pp. 159-160-, 26 (=AE 1962, 69) -pp. 162-164-, 28 (=HEp6, 117) -pp. 165-166-, 38 -pp. 177-179- o 43 (HEp6, 115) -pp. 184-185- respectivamente. 
dicado a Lucius Attius por Memmius Suau[is] y Memmia Su[a]uola (AE 1993, 910), o, con secuencias y estructuras onomásticas aun más parecidas a la de nuestra pieza, el que dedican Polytimus y Capitollina a Norbana Calephasis (CIL II, 58) o el que a Euhodia dedican sus conliberti Euhodius y Callityche (AE 1982, 485).

A partir de aqui, no resulta difícil, como se dijo, imaginar el resto de la estructura del texto dado lo estereotipado del formulario de estas estelas graníticas Emeritenses ${ }^{21}$. Como se dijo más arriba, serían esperables —en función de la metrologia de las letras y de la modulación del fragmento- al menos dos o tres líneas por encima de la primera conservada - para la indicación de la fórmula consecratoria $D$ (is) $M$ (anibus) s(acrum) y para la expresión del nombre del difunto y, acaso, de su edad - y la última línea del texto — siguiente a la última de las tres conservadas- parece legítimo pensar que incorporaría la fórmula dedicatoria final que, en función del hábito epigráfico emeritense, bien podría ser f(aciendum) $c$ (urauerunt) bien, en su defecto, sencillamente $f($ ecerunt), por más que esta propuesta no deje de ser una simple hipótesis interpretativa que no debería excluir otros finales como simplemente la indicatio pedaturae - in agr(o) p(edes) - o como la presencia de algún sustantivo de carácter familiar precediendo al verbo de la dedicación pudiendo, pues, con todo lo dicho, pensarse en una estructura semejante a ésta:

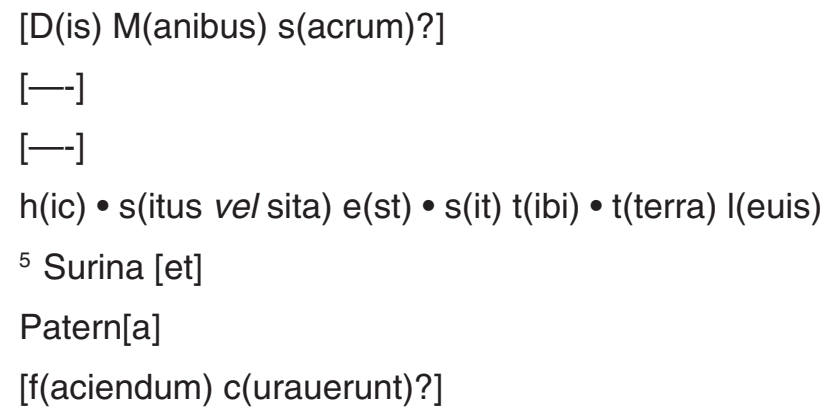

Desde el punto de vista cronológico y para la datación de la pieza, la incorporación —-precisamente- de la fórmula $h$ (ic) s(itus) e(st) s(it) t(ibi) t(erra) I(euis) necesariamente permite pensar en una fecha posterior a finales del siglo I d. C., tal vez ya del siglo II d. C., para nuestra inscripción coincidiendo, por tanto, con el esplendor de la producción epigráfica emeritense y, por supuesto, con la de este tipo de estelas funerarias graníticas ${ }^{22}$ con las que debe ponerse en relación este nuevo y generoso documento epigráfico.

${ }^{21}$ EDMONSON, Jonathan, op. Cit. (n. 5), pp. 82-88.

22 EDMONSON, Jonathan, op. Cit. (n. 5), pp. 79-80. 
